\title{
An empirical approach to the nucleation of sulfuric acid droplets in the atmosphere
}

\author{
Patrick Hamill $\left({ }^{1}\right)$, Raffaella D'Auria $\left({ }^{2}\right)$ and Richard P. Turco $\left({ }^{2}\right)$ \\ ( $\left.{ }^{1}\right)$ Department of Physics, San José State University, U.S.A. \\ $\left(^{2}\right)$ Department of Atmospheric Sciences, University of California, Los Angeles, U.S.A.
}

\begin{abstract}
We use quantum mechanical evaluations of the Gibbs free energy of the hydrates of sulfuric acid, $\mathrm{H}_{2} \mathrm{SO}_{4} \cdot n \mathrm{H}_{2} \mathrm{O}$ and $\left(\mathrm{H}_{2} \mathrm{SO}_{4}\right)_{2} \cdot n \mathrm{H}_{2} \mathrm{O}$ to evaluate an empirical surface tension for sulfuric acid-water clusters containing few molecules. We use this surface tension to evaluate nucleation rates using classical heteromolecular theory. At low temperatures $(T \simeq 213 \mathrm{~K})$ the nucleation rates obtained with the empirical surface tensions are significantly greater than those using bulk values of the surface tension. At higher temperatures the difference disappears.
\end{abstract}

Key words nucleation - Gibbs energy - aerosol microphysics

\section{Introduction}

The formation rate of sulfate particles in the atmosphere is usually evaluated using the «classical» theory of homogeneous, heteromolecular nucleation. This is based on the theory of the homogeneous nucleation of a single substance (usually water) as developed by Volmer and Weber (1926), Becker and Doring (1935), Frenkel (1946), and others. An overview of the theory was presented by McDonald $(1962,1963)$. The experimental verification of the theory was based on determining the value of the supersaturation when particle formation was observed to occur. For pure water, the theory agrees reasonably well with experiment, and for most substances the predicted values of super-saturation are typically accurate to within $10 \%$, but for other substances

Mailing address: Prof. Patrick Hamill, Physics Department, San José State University, San José, CA 95192, U.S.A.; e-mail: hamill@wind.sjsu.edu the theoretical predictions and experimental results differ significantly. Techniques that have been developed to actually count the number of particles formed in a nucleation process indicate that the dependence of nucleation rate on supersaturation is approximately correctly given by the classical theory but the temperature dependence is not (Oxtoby, 1992). The predicted nucleation rates are typically too low at low temperatures and too high at high temperatures, disagreeing with experimental values by several orders of magnitude.

The classical theory (as described below in Section 2) depends on the change in the Gibbs free energy of an embryonic droplet as molecules are added to it. The Gibbs free energy depends on the surface tension, a quantity that is poorly defined for clusters of a few molecules.

In this paper in Section 2 we give a brief review of the classical theory of heteromolecular nucleation, particularly as applied to the formation of sulfate particles in the atmosphere. As mentioned below, this theory severely underestimates observed environmental nucleation rates.

In Section 3 we consider the quantum mechanical calculations that have been carried out 
by Bandy and Ianni (1998) and Ianni and Bandy (2000) which allow one to determine the free energy increase of a hydrate of sulfuric acid as water molecules are added to it.

In Section 4 we combine the classical theory with the quantum mechanical results by introducing an empirical surface tension term such that the Gibbs free energy predicted by the classical theory roughly agrees with the value obtained from the quantum mechanical calculations. We then evaluate nucleation rates for sulfate particles using the empirical (size, composition and temperature dependent) surface tensions to determine the effect on nucleation rates. In Section 5 we present our conclusions.

\section{The classical theory of homogeneous nucleation}

\subsection{Nucleation of a single substance from the vapor}

It is convenient to begin by considering the homogeneous nucleation of a pure substance. The classical theory is based on the difference in the Gibbs free energy of $n$ free molecules of the condensing substance, and the Gibbs free energy of these molecules when they are incorporated into a droplet. When the molecules are in the droplet, they are assumed to be in the liquid phase, meaning that the Gibbs free energy has been reduced by a quantity $-n \ln (S)$ where $S=P / P^{0}$ is the supersaturation. Here $P$ is the partial pressure of the vapor and $P^{0}$ is the equilibrium vapor pressure of the liquid. The molecules that form a droplet embryo have not only undergone a phase change from vapor to liquid, they have also formed a sphere with a surface of area of $A=4 \pi r^{2}$. The energy associated with the construction of this surface is $\sigma A$ where $\sigma$ is the surface tension. Thus the change in Gibbs free energy when $n$ molecules are transformed from free vapor molecules to molecules in the liquid state in a droplet of area $A$ is

$$
\Delta G=-n k T \ln S+\sigma A
$$

where $k$ is Boltzmann's constant and $T$ is the temperature. If the density of the liquid is $\rho$ this expression can be written in terms of the radius of the droplet $r$ as follows:

$$
\Delta G=-\frac{4}{3} \pi r^{3} \rho R T \ln S+4 \pi r^{2} \sigma
$$

where $R$ is the gas constant per gram of vapor.

For $S>1$ the function $\Delta G$ passes through a maximum

$$
\Delta G^{*}=16 \pi \sigma^{3} / 3(\rho R T \ln S)^{2}
$$

at the «critical» embryo radius $r^{*}$ that satisfies the Kelvin equation

$$
r^{*}=2 \sigma / \rho R T \ln S .
$$

The surface energy term in eq. (2.2) increases with $r^{2}$ while the bulk energy term grows more negative as $r^{3}$. In general a plot of $\Delta G$ versus $r$ shows an increase to a maximum $\Delta G^{*}$ at $r^{*}$ and then a decrease to negative values. Note that $r$ is not a continuous variable because the particle grows by incorporating integral numbers of molecules. The functional form of eq. (2.2) implies that $\Delta G$ increases to a positive maximum before decreasing to negative values but since $n$ can only take on integer values (see eq. (2.1)), if the supersaturation $S$ is large enough $\Delta G$ may not exhibit a maximum at all.

In the classical theory one assumes that the vapor will contain clusters of molecules (embryos) which satisfy a Boltzmann relation

$$
n_{g}=n_{1} e^{-\Delta G / k T}
$$

where $n_{g}$ is the number of clusters containing $g$ molecules. The «critical» cluster contains $g^{*}$ molecules. Note that for a cluster with $g<g^{*}$ the addition of one more molecule implies an increase in $\Delta G$, but for a cluster with $g>g^{*}$ the addition of one more molecule leads to a decrease in $\Delta G$, and the embryo grows spontaneously.

The nucleation rate can be determined by evaluating the number of critically sized clusters and the net rate at which molecules are incorporated into them. One usually defines a «current» $J_{g}$ which is the net rate at which $g$-mers 
are formed by

$$
J_{g}=C_{g} n_{g}-E_{g+1} n_{g+1}
$$

where $C_{g}$ is the condensation rate of single molecules from $g$-mers and $E_{g+1}$ is the evaporation rate from $(g+1)$-mers.

It can be shown (McDonald, 1963, for example) that given several reasonable assumptions (including the assumption of a steady state situation so that $J_{g}$ is a constant independent of $g$ ), this current or nucleation rate, can be expressed as

$$
J=\left(n^{2} / \rho\right)(2 \sigma m / \pi) e^{-\Delta G^{*} / k T} .
$$

Here $n$ is the number density of molecules in the vapor, related to the pressure by $p=n k T$.

Considering the form of $\Delta G^{*}$ given by eq. (2.3), we appreciate the importance of the surface tension in the nucleation rate calculation as $J$ depends on the surface tension cubed in an exponential.

\subsection{Heteromolecular nucleation}

By heteromolecular nucleation we mean the nucleation of liquid drops from a vapor containing two or more substances. We shall limit ourselves to the case of binary system nucleation of solution droplets of sulfuric acid and water.

It is well known that solution droplets can form under conditions in which neither one of the pure substances could form. This is because the vapor pressure of a component in a binary solution is less than the vapor pressure of the pure substance.

Heteromolecular nucleation (sometimes referred to as «binary nucleation» since there are usually only two components participating in the process) was studied by Doyle (1961), Reiss (1950), Heist and Reiss (1974), Kiang and Stauffer (1973), Jaecker-Voirol and Mirabel (1989), Hamill et al. (1977) and many others. This is a straightforward generalization of the homogeneous «monomolecular» nucleation theory described above. However, it should be mentioned that the theory originally developed by Doyle (1961) and others suffers from a thermodynamic inconsistency in the evaluation of the critical value of the Gibbs free energy increase. This has led to a «corrected» theory by Wilemski (1984) and others.

We shall consider nucleation theory as applied to the formation of a solution droplet with two components labelled $a$ (acid) and $w$ (water). In this case the Gibbs free energy change is given by

$$
\Delta G=-n_{a} k T \ln S_{a}-n_{w} k T \ln S_{w}+4 \pi r^{2} \sigma
$$

where $S_{a}=P_{a} / P_{a}^{0}$ is the supersaturation of component $a$, defined as the partial pressure of component $a$ in the environment divided by the equilibrium vapor pressure of component $a$ over a flat surface having the same composition as the solution, and similarly for $S_{w}$.

The nucleation rate is then given by

$$
J=4 \pi r^{* 2} \beta_{a} N_{w} \exp \left(-\Delta G^{*} / k T\right)
$$

where $N_{w}$ is the number density of $w$ molecules in the environment and $\beta$ is the rate at which $a$ molecules impinge upon the embryonic droplet of radius $r$.

In the case of a binary system, the Gibbs free energy is a function of $n_{a}$ and $n_{b}$ and consequently we must consider a Gibbs free energy surface. The critical values ( $r^{*}$ and $\Delta G^{*}$ ) correspond to a saddle point on this surface. That is, the $\Delta G$ surface can be envisioned as a mountain range with a pass through it. The highest point in the pass is the critical value. However, at high enough values of supersaturation the Gibbs free energy surface will have no barrier to nucleation.

In fig. 1 we present the Gibbs free energy surface using bulk values of surface tension and density. This figure illustrates the $\Delta G$ surface for the number of acid molecules ranging up to five and the number of water molecules ranging up to ten. The critical size is at about 3 acid molecules and 6 water molecules. The calculation was carried out assuming a temperature of $240 \mathrm{~K}$, an environmental pressure of $125 \mathrm{mb}$, a relative humidity of $50 \%$ and a sulfuric acid concentration of $5 \mathrm{ppt}$, or about $1.9 \times 10^{7}$ molecules $/ \mathrm{cm}^{3}$. This concentration, while common in the lower troposphere, represents an extreme value in upper tropospheric conditions (e.g., Clarke et al., 1999). For these conditions the nucleation rate predicted 


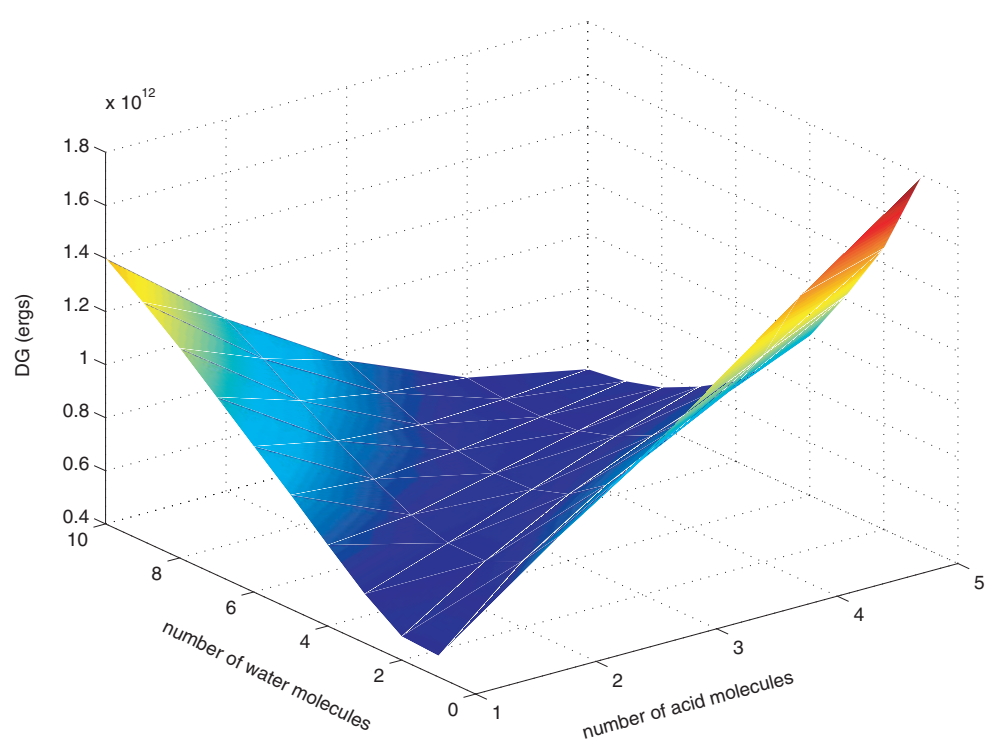

Fig. 1. The $\Delta G$ surface as a function of the number of sulfuric acid molecules and the number of water molecules in the cluster, assuming classical theory. The environmental conditions assumed in the calculation are a temperature of $240 \mathrm{~K}$, an environmental pressure of $125 \mathrm{mb}$, a relative humidity of $50 \%$ and a sulfuric acid concentration of 5 ppt. The critical size is the saddle point in this «pass» through the $\Delta G$ barrier. It is located at $n_{a}=3$ and $n_{w}=6$.

by eq. (2.9) is $10^{13}$ droplets $/ \mathrm{cm}^{3} \cdot \mathrm{s}$, which is, of course, unreasonably high since it predicts that the number of droplets formed per second is six orders of magnitude larger than the total number of available sulfuric acid molecules.

If, however, we include the effect of hydrates as suggested by Heist and Reiss (1974) and Jaecker-Voirol et al. (1987) the nucleation rate for these conditions drops to 4.6 droplets $/ \mathrm{cm}^{3} \cdot \mathrm{s}$. The predicted saddle point is at 4 sulfuric acid molecules and 13 water molecules. The results of calculations such as this agree relatively well with those presented by Laaksonen and Kulmala (1991). Those investigators present a table of results including the number of water and acid molecules in a critical cluster and in the majority of the cases given the values of $n_{a}$ and $n_{b}$ are less than ten. Our nucleation rates differ from theirs by a factor of about 10 due to the fact that our expression for the prefactor to the exponential does not take into account the effect of hydrates.
It is clear, then that the theory of heteromolecular nucleation for sulfuric acid water solution droplets involves very small clusters of just a few molecules. Using macroscopic concepts of density and surface tension require extrapolations which are very hard to justify.

A particularly serious problem with the theory is that it does not predict the formation of new particles under conditions in which new particle formation is observed to occur (O'Dowd, 2001; Kulmala et al., 1998). However, Clarke et al. (1999) found that particle formation in the upper troposphere is often in agreement with predictions of classical binary nucleation theory if the effect of hydrates is ignored.

\section{Quantum mechanical calculations}

Recently, Bandy and Ianni (1998) and Ianni and Bandy (2000) carried out density functional theory quantum mechanical calculations of the 
hydrates of sulfuric acid. They evaluated the Gibbs free energy increase in the reactions

$\mathrm{H}_{2} \mathrm{SO}_{4} \cdot(n-1) \mathrm{H}_{2} \mathrm{O}+\mathrm{H}_{2} \mathrm{O} \rightarrow \mathrm{H}_{2} \mathrm{SO}_{4} \cdot n \mathrm{H}_{2} \mathrm{O}$

and

$$
\left(\mathrm{H}_{2} \mathrm{SO}_{4}\right)_{2} \cdot(n-1) \mathrm{H}_{2} \mathrm{O}+\mathrm{H}_{2} \mathrm{O} \rightarrow\left(\mathrm{H}_{2} \mathrm{SO}_{4}\right)_{2} \cdot n \mathrm{H}_{2} \mathrm{O} .
$$

Bandy and Ianni (1998) considered reaction (3.1) using the «Gaussian 94» program for their calculations. An interesting result of the study was the fact that although the bulk sulfuric acid-water solution is highly ionized, the clusters of a few molecules did not exhibit ionization until $n=7$. The motivation for the Bandy and Ianni (1998) study was the fact that the hydrates of sulfuric acid are thought to play an important role in the formation of new atmospheric particles. They point out that previous studies have assumed that the properties of small clusters of sulfuric acid and water could be obtained by extrapolating the properties of the bulk phase. They found that for a temperature of $298 \mathrm{~K}$, reaction (3.1) has Gibbs free energies that are slightly negative for $n=1-3$ and slightly positive for $n=4-7$ with a maximum at $n=5$. For lower temperatures, the Gibbs free energies decrease. They computed equilibrium constants and found that the values estimated by Jaecker-Voirol et al. (1987) were orders of magnitude larger than predicted by the density functional theory results. Bandy and Ianni (1998) suggest that hydrates play a much smaller role in homogeneous nucleation theory than previously believed. It has been supposed that the clustering of water molecules around sulfuric acid molecules would greatly reduce the number of free water and sulfuric acid molecules in the environment. Therefore, in this paper we have not included hydrates as a constituent of the environmental vapor in any of our nucleation calculations.

Ianni and Bandy (2000) carried out a study of the hydrates of $\left(\mathrm{H}_{2} \mathrm{SO}_{4}\right)_{2}$. They found that all the higher hydrates of $\left(\mathrm{H}_{2} \mathrm{SO}_{4}\right)_{2}$ linearly decrease the free energy of the system. They developed a kinetic model of the growth of the hydrates and concluded that although $\left(\mathrm{H}_{2} \mathrm{SO}_{4}\right)_{2} \cdot n \mathrm{H}_{2} \mathrm{O}$ with $n=0-6$ will spontaneously form from $\mathrm{H}_{2} \mathrm{SO}_{4} \cdot \mathrm{H}_{2} \mathrm{O}$ it seems to be kinetically and thermodynamically prohibited. They suggest that perhaps a third species such as ammonia or the formation of an excited state is required to stabilize the system and allow for larger hydrate chains to grow. In our analysis we have ignored these problems, and perhaps future quantum mechanical studies will answer some of these questions.

\section{Evaluation of empirical surface tensions and nucleation rates}

In view of the fact that the surface tension is the most important parameter determining the Gibbs free energy increase and hence the nucleation rate, we used the studies of Bandy and Ianni (1998) to obtain empirical values of surface tension for very small droplets. For reasons mentioned below, we believe that the bulk value of surface tension is valid to very small droplets, but fails for clusters containing less than about seven molecules. This would not be important if it were not for the fact that the critical size of sulfuric acid-water solution droplets is extremely small, as mentioned in Section 1.

The reason why we believe that the bulk surface tension is an accurate description of the surface tension of very small droplets is based on a study carried out by D'Auria (2001) in which the quantum mechanical evaluations of the Gibbs free energy change for water molecules clustered about a hydronium ion were compared with evaluations obtained from the classical theory. Figure 2 shows this comparison. Note that even for clusters as small as 6 or 7 molecules the classical theory agrees very well with the quantum mechanical simulations. If one were to define a surface tension for the cluster which increases with number of molecules in the cluster, and reaching the bulk value for (say) 7 molecules, then one could fit the classical theory to the quantum mechanical results.

This is what we have done for the binary solution, sulfuric acid and water. Of course the system is more complicated, being composed of two substances. Furthermore, the quantum mechanical studies are limited to the two processes described by eqs. (3.1) and (3.2).

In fig. 3 we show the quantum mechanically evaluated values for $\Delta G$, taken from Bandy and 
Patrick Hamill, Raffaella D’Auria and Richard P. Turco

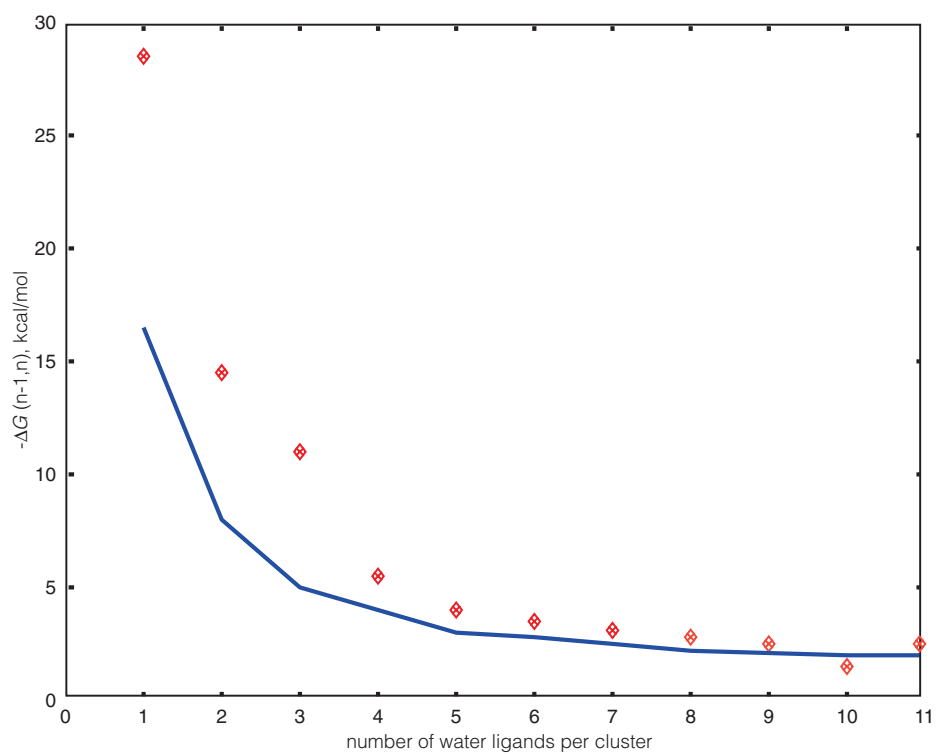

Fig. 2. A comparison between the Gibbs free energy change associated with the hydronium/water clustering reaction as obtained from the classical model and the quantum mechanical simulations. The solid line is for the classical model and the symbols are the quantum mechanical calculated results (from D'Auria, 2001).
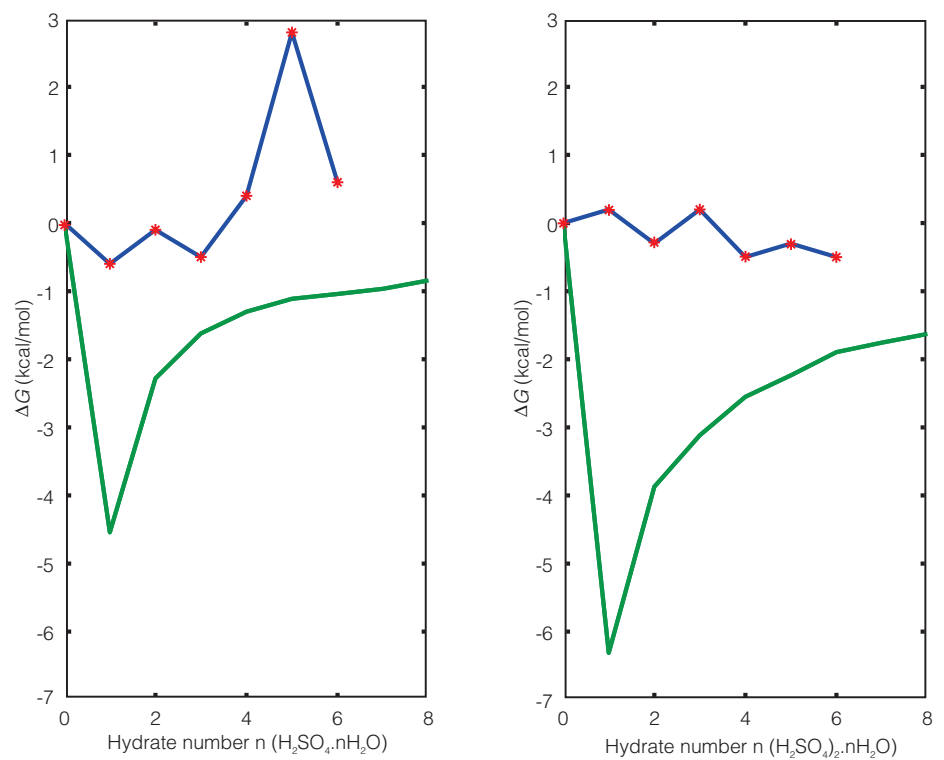

Fig. 3. The change in Gibbs free energy due to adding a water molecule to $\mathrm{H}_{2} \mathrm{SO}_{4} \cdot n \mathrm{H}_{2} \mathrm{O}$ (left panel) and to $\left(\mathrm{H}_{2} \mathrm{SO}_{4}\right)_{2} \cdot n \mathrm{H}_{2} \mathrm{O}$ (right panel). The top curves (with asterisks) are the quantum mechanical results and the bottom curves are the liquid droplet model results. 

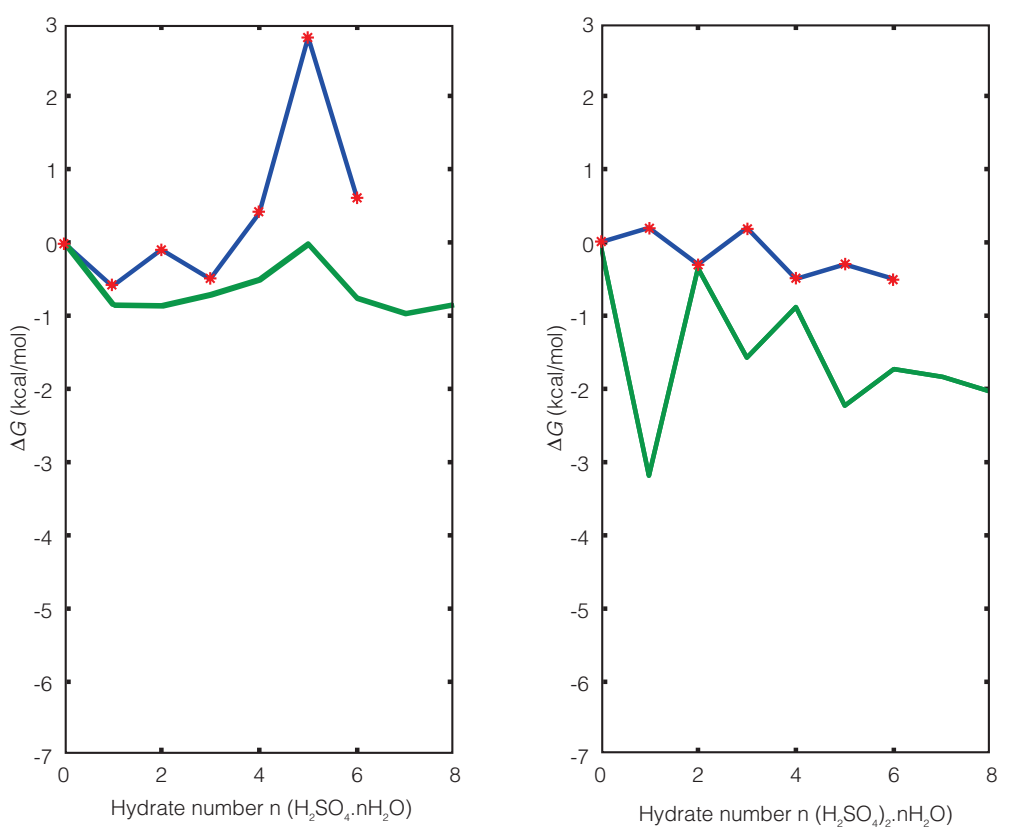

Fig. 4. The same as fig. 3 except that the surface tensions used in the classical calculation have been changed to get a closer agreement with the quantum mechanical results.

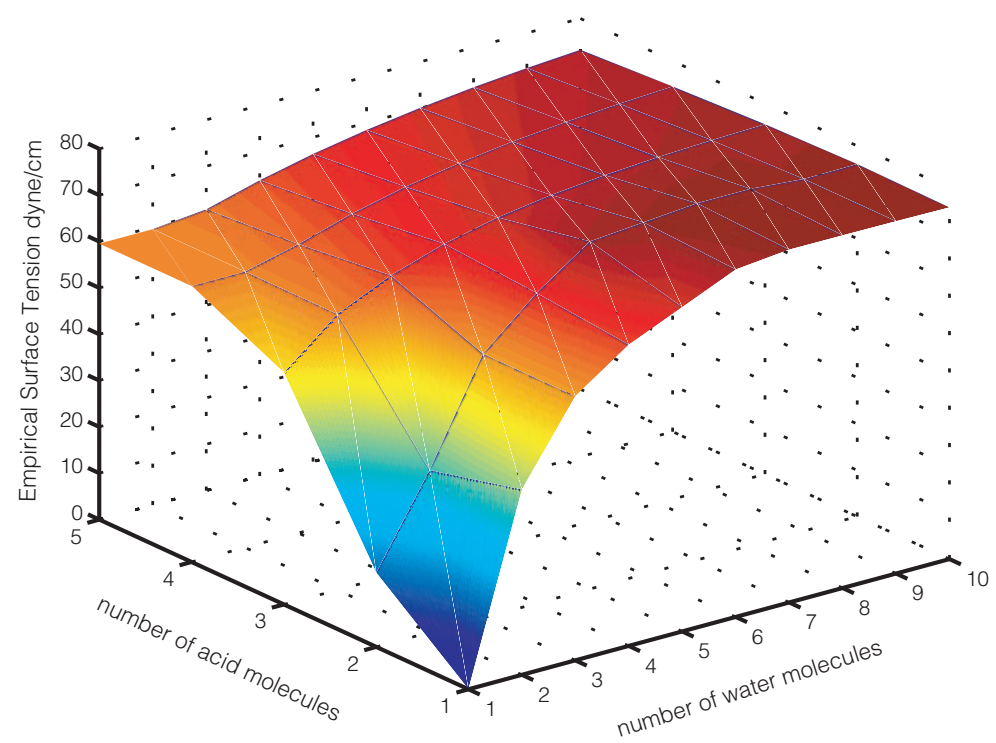

Fig. 5. The surface tension that was used to generate the classical curves of fig. 4, presented in terms of the number of water and sulfuric acid molecules in the embryonic droplet. 
Ianni (1998) and Ianni and Bandy (2000), and the values obtained from the classical theory, eq. (2.8). The curves show the change in Gibbs free energy due to adding a water molecule to $\mathrm{H}_{2} \mathrm{SO}_{4} \cdot n \mathrm{H}_{2} \mathrm{O}$ (left panel) and to $\left(\mathrm{H}_{2} \mathrm{SO}_{4}\right)_{2} \cdot n \mathrm{H}_{2} \mathrm{O}$ (right panel). The top curves (with asterisks) are the quantum mechanical results and the bottom curves are the liquid droplet model results.

It is not entirely clear from the figure at what point the two curves will come together, that is, we cannot predict the size of a cluster at which the classical model agrees well with the quantum mechanical calculation. However, guiding our intuition by the plot in fig. 2 we shall assume that clusters with about 10 water molecules will be reasonably described by the classical model.

The classical curves can be made to approximate the quantum mechanical curves by selecting appropriate choices of surface tension. In fig. 4 we present the curves of fig. 3 except

Table I. Empirical surface tension of cluster (dynes $\left./ \mathrm{cm}^{2}\right)$.

\begin{tabular}{ccccccccccc}
\hline \hline $\mathrm{H}_{2} \mathrm{O}$ & 1 & 2 & 3 & 4 & 5 & 6 & 7 & 8 & 9 & 10 \\
\hline $\mathrm{H}_{2} \mathrm{SO}_{4}$ & & & & & & & & & & \\
\hline 1 & 0 & 40.0 & 57.0 & 65.0 & 70.0 & 75.0 & 76.2 & 76.1 & 76.0 & 75.9 \\
2 & 16.0 & 35.0 & 57.0 & 67.0 & 75.0 & 76.0 & 77.0 & 77.0 & 76.0 & 76.1 \\
3 & 50.0 & 59.6 & 64.8 & 68.5 & 71.0 & 72.7 & 73.9 & 74.7 & 75.2 & 75.5 \\
4 & 59.6 & 59.6 & 62.3 & 65.8 & 68.5 & 70.5 & 72.1 & 73.1 & 73.9 & 74.5 \\
5 & 59.6 & 59.6 & 60.7 & 63.8 & 66.4 & 68.5 & 70.1 & 71.4 & 72.5 & 73.3 \\
\hline
\end{tabular}

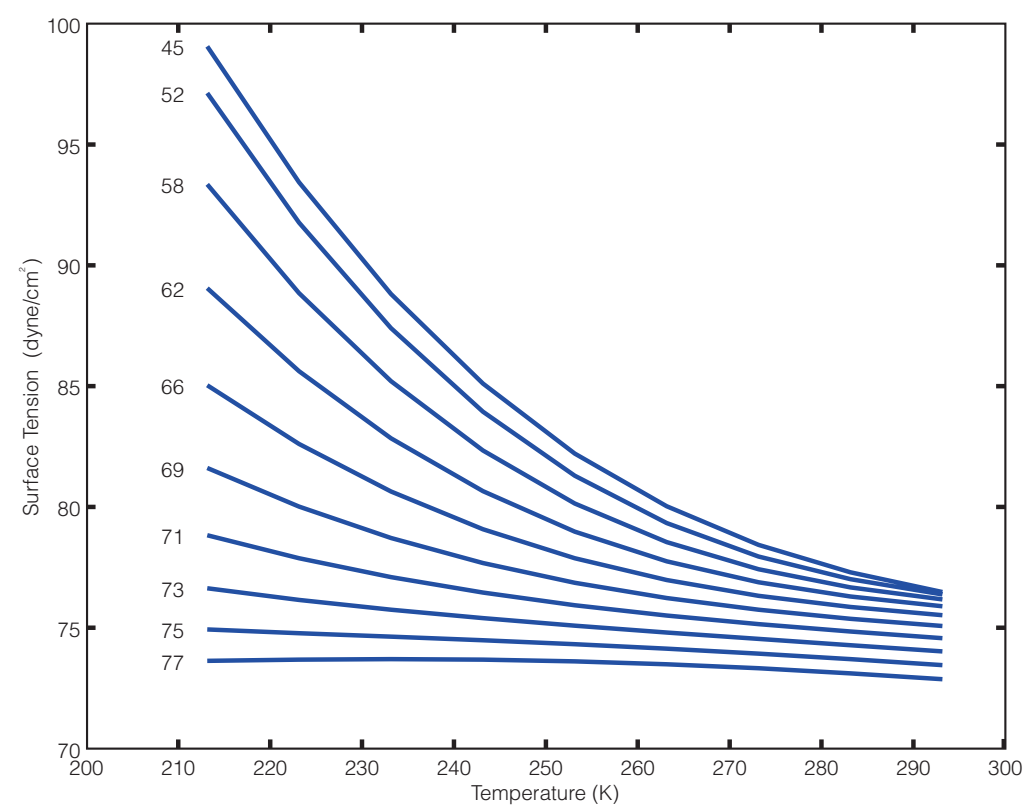

Fig. 6. Surface tension as a function of temperature for a bulk sulfuric acid water solution. The numbers on each curve represent the weight percentage sulfuric acid. 

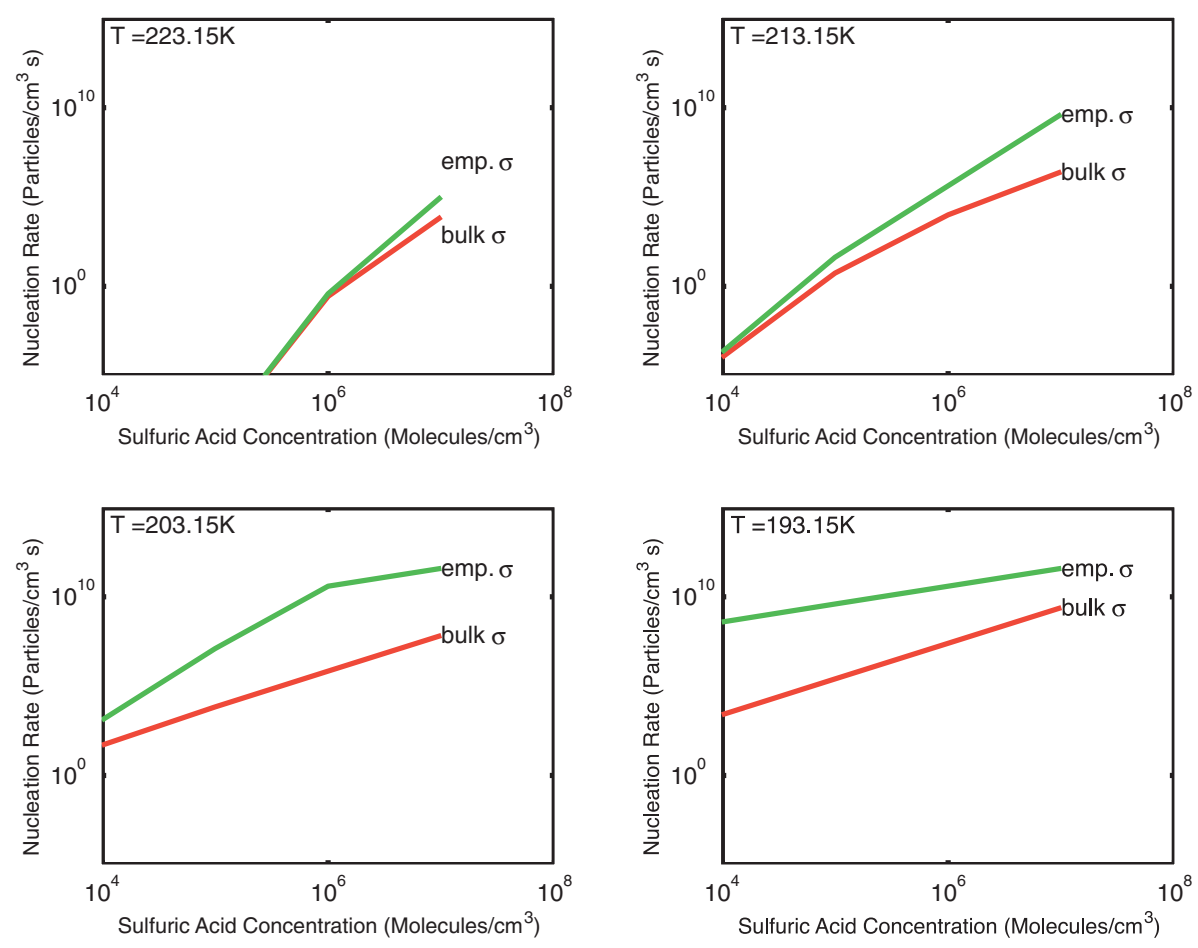

Fig. 7. Nucleation rate versus sulfuric acid concentration using the empirically obtained surface tension compared to the bulk surface tension for various values of the temperature. In all cases the water concentration was assumed to be $10^{13}$ molecules $/ \mathrm{cm}^{3}$.

that in evaluating the classical Gibbs free energy difference we used surface tension values that brought these curves closer to the quantum mechanical values. We could, of course, have fit the quantum results exactly, but then the surface tension would not have been a smoothly varying function. The surface tension used in fig. 4 is presented as a function of composition in fig. 5.

The surface tensions used are presented in table I as a function of the number of sulfuric acid molecules and the number of water molecules in the cluster.

In fig. 6 we show how the surface tension of a bulk solution of sulfuric acid and water varies as a function of temperature and composition. The numbers on each curve are the weight percentage of sulfuric acid in the solution.

In our calculations of the nucleation rate using the extrapolations of the surface tensions of table I and fig. 5 we assumed the same temperature dependence as in fig. 6. We find, as would be expected, that decreasing the surface tension increases the nucleation rate. In fig. 7 we illustrate the increase in nucleation rates obtained by using the empirical surface tensions rather than the bulk values. The figure presents nucleation rates as a function of sulfuric acid concentration using the empirically obtained surface tension and using the tension compared to the bulk surface tension for various values of the temperature. In all cases the water concentration was assumed to be $10^{13}$ molecules $/ \mathrm{cm}^{3}$. At the highest temperature $(223.15 \mathrm{~K})$, the nucleation rates are low and the change in the surface tension makes essentially no difference, except for the highest sulfuric acid concentrations $\left(10^{7}\right.$ molecules $/ \mathrm{cm}^{3}$ ). However, for lower temperatures the difference in nucleation rates is quite sig- 
nificant. For example, at $203 \mathrm{~K}$ (a temperature often experienced near the tropical tropopause) and a sulfuric acid concentration of $10^{6}$ sulfuric acid molecules $/ \mathrm{cm}^{3}$, the difference in nucleation rates is about five orders of magnitude.

\section{Conclusions}

We have shown that quantum mechanical calculations allow one to define an empirical surface tension for sulfuric acid water calculations. We see that these empirical surface tensions lead to greatly enhanced nucleation rates at lower temperatures. Our analysis is based on the two studies of the sulfuric acid-water system carried out by Bandy and Ianni (1998) and Ianni and Bandy (2000). These studies involved reactions (3.1) and (3.2) and therefore only include clusters whose compositions are $\mathrm{H}_{2} \mathrm{SO}_{4} \cdot n \mathrm{H}_{2} \mathrm{O}$ and $\left(\mathrm{H}_{2} \mathrm{SO}_{4}\right)_{2} \cdot n \mathrm{H}_{2} \mathrm{O}$. Therefore, we cannot generate empirical surface tensions for all the compositions that are included in the $\Delta G$ surface illustrated in fig. 1. Consequently, our results are incomplete. For this reason we have not attempted to refine our method of determining the empirical surface tensions; such work will be carried out when the quantum mechanical results are available.

Our main conclusion is, then, that cluster surface tensions can be defined and can be used to calculated nucleation rates. The nucleation rates obtained are higher than predicted by using the bulk values of surface tension.

\section{Acknowledgements}

This work has been funded by the NSF under grant ATM-00-70847, and NASA under grant NAG1-1899. RD is also supported by NASA Earth System Science Fellowship ESS/ 00-0000-0080.

\section{REFERENCES}

BANDY, A.R. and J.C. IANNI (1998): Study of the hydrates of $\mathrm{H}_{2} \mathrm{SO}_{4}$ using density functional theory, J. Phys. Chem. A, 102, 6533-6539.
BECKER, R. and W. DORING (1935): Kinetische behandlung der keimbildung in übersättigten dampfern, Ann. Phys., 24, 719-752.

Clarke, A.D., V.N. Kapustin, F.L. Eisele, R.J. Weber and P.H. MCMURRY (1999): Particle production near marine clouds: sulfuric acid and predictions from classical binary nucleation, Geophys. Res. Lett., 26, 2425-2428.

D'AURIA, R. (2001): A study of ionic clusters in the middle and lower atmosphere and their role in aerosol formation, a thesis topic proposal (unpublished ms.).

DOYLE, G.J. (1961): Self-nucleation in the sulfuric acid-water system, J. Chem. Phys., 35, 795-799.

FRENKEL, J. (1946): The Kinetic Theory of Liquids (Clarendon Press, Oxford), pp. 488

HAmill, P., C.S. KIANG and R.D. CADLE (1977): The nucleation of $\mathrm{H}_{2} \mathrm{SO}_{4}-\mathrm{H}_{2} \mathrm{O}$ solution aerosol particles in the stratosphere, J. Atmos. Sci., 34, 150-162.

HEIST, R.H. and H. REISS (1974): Hydrates in supersaturated binary sulfuric acid-water vapor, J. Chem. Phys., 61, 573-581.

IANNI, J.C. and A.R. BANDY (2000): A theoretical study of the hydrates of $\left(\mathrm{H}_{2} \mathrm{SO}_{4}\right)_{2}$ and its implications for the formation of new atmospheric particles, J. Mol. Struct. Theochem, 497, 19-37.

JAECKER-VOIROL, A. and P. MIRABEL (1989): Heteromolecular nucleation in the sulfuric acid - water system, Atmos. Environ., 23, 2053-2057.

JAECKER-Voirol, A., P. Mirabel and H. Reiss (1987): Hydrates in supersaturated binary sulfuric-acid water vapor: a reexamination, J. Chem. Phys., 87, 4849-4852.

KIANG, C.S. and D. STAUfFER (1973 ): Chemical nucleation theory for various humidities and pollutants, Faraday Sym., 7, 26-33.

Kulmala, M., A. LaAksonen and L. Pirjola (1998): Parameterizations for sulfuric acid/water nucleation rates, J. Geophys. Res., 103, 8301-8307.

LAaksonen, A. and M. Kulmala (1991): Homogeneous heteromolecular nucleation of sulphuric acid and water vapours in stratospheric conditions: a theoretical study of the effect of hydrate interaction, J. Aerosol Sci., 22, 779-787.

MCDONALD, J.E. (1962): Homogeneous nucleation of vapor condensation. I. Thermodynamic aspects, Am. J. Phys., 30, 870-877.

MCDONALD, J.E. (1963): Homogeneous nucleation of vapor condensation. II. Kinetic aspects, Am. J. Phys., 31,31-41.

O'DowD, C. (2001): Advances in elucidating new particle formation in the marine and coastal boundary layers, in European Geophysical Society 26th General Assembly, Nice, 2001, Geophys. Res. Abstr., 3, 4794.

OXTOBY, D.W. (1992): Homogeneous nucleation: theory and experiment, J. Phys. Condensed Matter, 4, 7627-7650.

REISS, H. (1950): The kinetics of phase transitions in binary systems, J. Chem. Phys, 18, 840-848.

Volmer, M. and A. WeBer (1926): Keimbildung in übersättigten Gebilden, Z. Phys. Chem., 119, 277-301.

WILEMSKI, B. (1984): Composition of the critical nucleus in multicomponent vapor nucleation, J. Chem. Phys., 80, 1370-1372. 